
DE DONATO GIANNOTTI (1492-1573)

\author{
Gérard VITTORI \\ Université de Rennes 2 (France)
}

\begin{abstract}
La comédie Le Vieillard amoureux met en scène une situation de désordre : deux pères en rivalité amoureuse avec leurs deux fils pour la possession d'une jeune fille. Les deux vieillards veulent abuser de leur autorité de pères ; mais c'est le contrôle exercé par les tiers, par le regard et le discours qu'ils profèrent ou sont susceptibles de proférer, qui contribue au dénouement de la pièce. Nous envisageons le fonctionnement de ce contrôle social comme discours de régulation des rapports humains.
\end{abstract}

Mots-clés: Donato Giannotti ; Discours ; Autorité ; Pouvoir ; Exclusion ; Désordre ; René Girard

\title{
Social control in Il vecchio amoroso by Donato Giannotti (1492-1573)
}

The comedy The old Man in Love depicts a situation of disorder: two fathers-in-law compete with their sons for the love of a young girl. The two old men want to abuse their fatherly authority but the control exercised by the third parties, by look and by the speech they utter or are likely to utter, does contribute to the outcome of the play. We regard the function of this discourse as social control aiming to regulate human relationships

Key words: Donato Giannotti ; Speech ; Authority ; Power ; Exclusion ; Disorder; René Girard

La censure, au sens général du terme, est une modalité de la relation politique entre un pouvoir et les créateurs ; elle est une entrave à la liberté d'expression. Elle est la manifestation la plus crue d'un rapport de force qui s'exerce en faveur de ceux qui détiennent les rouages d'une nation. Ceci ne signifie pas qu'en dehors de la censure - et celle-ci ôtée -, chacun puisse agir, dans les formes de sa vie quotidienne, sans aucune contrainte. Il existe un pouvoir diffus qui pèse sur les individus, les astreint à certains comportements ou les soumet à certains interdits. Il n'existe alors pas nécessairement une instance extérieure à la communauté : la cohésion des groupes humains s'appuie sur une auto-régulation interne, où chacun tour à tour peut exercer sur autrui un pouvoir de contrôle, de rappel à l'ordre, ou de condamnation. Il s'agit dans ce cas d'une censure plus diffuse qui peut éventuellement porter sur l'exercice de la parole, mais qui s'applique surtout aux actes pour assurer leur conformité aux règles et aux exigences ressenties comme vitales par le groupe humain au sein duquel ils se font jour. Dans une telle perspective, il est possible de proposer un élargissement de la notion de censure à celle de contrôle social, dont on peut voir un exemple de fonctionnement dans $L e$ Vieillard amoureux (Il vecchio amoroso) de Donato Giannotti. Il nous faudra alors préciser les points suivants : quel désordre justifie la mise en œuvre du contrôle social ? Quels sont les modes d'exercice ou les relais de ce contrôle social? De quels effets réels est-il porteur, c'est-à-dire par quelles contradictions de ceux qui exercent l'autorité le contrôle social peut-il voir atténués sa rigueur ou ses excès ? 


\section{Désordre et contrôle social comme tercéité}

Le contrôle social est habituellement mis en œuvre pour corriger, entre autres, un désordre qui peut être né d'un fait imprévu ou imprévisible. Le désordre dont il s'agit dans Il vecchio amoroso est l'amour qui prend naissance, dans l'esprit d'un vieillard, Teodoro, pour la jeune fille que son fils ramène comme domestique, tout au moins selon la présentation initiale qui est faite de la jeune personne. Cet aspect de la situation est livré dans le titre même de la pièce, non pas certes dans son détail, où il est fait état d'une anomalie, ou d'une sorte d'aberration prêtant au moins à sourire. Toutefois, la bienveillance présente dans le sourire, et la complicité qu'elle peut sous-entendre, ne sont pas seulement des marques de connivence avec le protagoniste; il y a aussi une complicité de ceux qui se gaussent d'un tiers, qui est détachement intérieur, distance à l'égard de cette part de soi qui reconnaît - ou pourrait reconnaître - comme sienne la « faiblesse » d'un autre. Le sourire est le premier degré du contrôle social qui, tout en laissant les actes s'accomplir et en ne retirant aucune autonomie au sujet, dissuade toute contagion chez ceux qui précisément portent un regard amusé sur un comportement qu'au fond ils comprennent, auquel ils accordent, à titre individuel, une légitimité humaine, mais dont ils nient la légitimité sociale. $\mathrm{Ce}$ 《sourire» représente une sorte de clivage entre l'aspect individuel et l'aspect social des actes, avec une option déjà marquée pour la prépondérance de la signification sociale des actes.

Le désordre que nous évoquons apparaît toutefois sur le fond d'un désordre général, marqué par une forme fortuite et par une forme rituelle : la forme fortuite du désordre, c'est la peste dans la ville dont revient le bateau ramenant à Pise Lionetto et la jeune esclave qu'il s'est promis d'épouser ${ }^{1}$. D'une façon symbolique, ce bateau mis en quarantaine représente le danger d'une contamination par le mal, le danger également d'une contamination impossible à contenir. Le fait que Lionetto, par un arrangement personnel avec le capitaine du port, ait pu entrer dans la ville, le fait que la jeune esclave et le domestique de Lionetto aient pu eux aussi descendre à quai (même si Lionetto ne les introduit pas directement dans la ville ${ }^{2}$ ) représente à la fois une exception et l'introduction non pas du désordre à proprement parler, mais de l'occasion pour ce dernier d'apparaître dans la communauté. Par un significatif renversement de situation, Lionetto avait dû quitter Pise, victime de la calomnie ${ }^{3}$ : un amour, pourtant tout à fait honorable, avait donné naissance - ou, plus exactement, prétexte - à des médisances qui étaient parvenues à son père comme d'incessants désordres, de la

\footnotetext{
${ }^{1}$ Cf. Lone Klem, La commedia del Cinquecento tra immagine del mondo reale e astrazione teatrale. Quattro esempi d'una dialettica.

<http://italiensk.au.dk/fileadmin/www.italiensk.au.dk/publikationer/atti_tutto_formato_pdf.pdf\#page $=106>$

Cet article analyse les sources classiques de la pièce. La bibliographie critique sur cette pièce de Giannotti est très réduite, presque inexistante. Au mieux, la pièce est mentionnée, sans plus. On pourra lire une analyse assez détaillée, écrite dans l'optique la genèse des œuvres de Goldoni dans M. Olsen, Goldoni et le drame bourgeois, 1995.

<http://diggy.ruc.dk/bitstream/1800/3704/1/Olsen_Goldoni.pdf>

${ }^{2}$ Son domestique et l'esclave demeurent dans une auberge par crainte de la peste.

${ }^{3}$ La calomnie a un mode de diffusion qui en fait, à l'instar de la peste, une contagion.
} 
débauche, du jeu et des dépenses sans contrôle. La raison économique avait fourni le juste motif d'un départ contraint qui mettrait fin aux rumeurs en abolissant leur source. Ce départ, l'abandon d'une relation n'ayant pourtant rien de coupable, ont mis un terme au discours circulant sur le compte de Lionetto :

«A Palerme, j'ai laissé de moi un très bon souvenir; et ici j'ai trouvé à mon retour la faveur de tous, et une bonne compréhension de ma situation. $»^{4}$

Lionetto, après un revers de fortune symbolique, peut se réjouir de l'état de l'opinion à propos de lui : il n'y est en fait pour rien, dans aucun sens, négatif ou positif. C'est une donnée de la situation, c'est un fait à accepter tel qu'il est, tel qu'il se présente, sans que quoi que ce soit puisse y être changé. Mais au moment de son retour, cette relation universellement positive (à Pise comme à Palerme) qui caractérise l'opinion des autres est sans rapport avec la réalité des choses: Lionetto revient avec une esclave tunisienne qu'il a rendue enceinte, qu'il aime et qu'il veut épouser. Voilà bien un désordre réel, qui, s'il n'est pas apparu à Palerme, ne devrait pas tarder à éclater au grand jour, provoquant un réel scandale. Mais pour l'heure, là où est un désordre réel, on trouve une relation positive de la communauté à l'égard de Lionetto, situation exactement inverse de celle qui existait au moment de son départ forcé de Pise. Cependant, par delà tout ceci, il est un désordre plus réel encore : la rivalité du père avec le fils. La relation de rivalité habituelle qui oppose le fils au père est en quelque sorte un désordre intégré : il fait partie de la vie de toute communauté, et il comporte une limite infranchissable, probablement fondatrice de toute vie sociale, la rivalité pour la possession d'une femme élue parmi toutes, la mère. Ce désordre-là étant évité ${ }^{5}$, c'en est un autre qui naît, tout aussi grand : la rivalité du père avec son fils. L'ami du père, Teodoro, en fait expressément la remarque : «- Si je comprends bien, tu es le rival de ton fils. » ${ }^{6}$ Il s'agit bien là d'un désordre, et à double titre : tout d'abord parce que toute rivalité, en tant que telle et par sa capacité de contagion mimétique, est source de désordre, et ensuite parce que nous sommes là face à un renversement de la situation habituelle de rivalité, à une inversion de son orientation «normale ». Il convient toutefois de préciser que la rivalité présente un double aspect au regard de sa relation au désordre : celui que nous venons de souligner (source virtuelle), mais aussi, à l'inverse, celle de contrôle social. Ainsi, lorsque deux femmes voisines, Gostanza et Cecca, voient arriver deux beaux jeunes hommes (Lionetto

\footnotetext{
${ }^{4}$ Il vecchio amoroso, in Borsellino, N. (a cura di), Commedie del Cinquecento, Milano, Feltrinelli, 1962, pp. 11-12. Nous noterons cette édition VA (Il vecchio amoroso) pour toutes nos citations.

${ }^{5}$ L'obligation de Lionetto de partir est peut-être liée à une relation à caractère scandaleux avec Giulia Bolognese, tunisienne. Le fait que cette jeune fille ou cette femme (l'âge n'est pas précisé, et le scandale pourrait tenir au caractère incestueux de la relation entre un jeune homme et une femme bien plus âgée que lui, et en outre tunisienne ; ce caractère quasi incestueux ne serait que le prélude inversé de l'autre désir quasi incestueux de relation de son père avec la jeune femme que Lionetto a ramenée ; nous traiterons cet aspect des choses plus avant). Lionetto semble avoir une constante dans ses goûts et son attirance pour des femmes d'ailleurs. Qu'il introduise dans sa communauté d'origine des femmes venues de l'extérieur n'est pas sans introduire une forme de désordre (cf. l'étranger dans les travaux de René Girard).

${ }^{6}$ VA, p. 26.
} 
et son ami Panfilo), une rivalité se déclenche immédiatement entre elles. Il est vrai que les circonstances sont particulières, et que l'invitation au désir, à un désir hors de tout code, est suscitée par la période de carnaval : tout le monde y paraît jeune, le désir de chacun est vivifié, l'ordre habituel est rituellement subverti et tout semble possible. Les masques que portent les hommes confèrent l'anonymat, c'est-à-dire l'impossibilité pour les autres de les situer dans le maillage du code social. Le désir peut circuler librement, les flux ne circulent plus selon des canaux déjà constitués. Mais au sein de cette liberté, virtuellement totale, il existe tout de même une régulation, inhérente à la convoitise de l'objet, qui est la convoitise de deux êtres différents pour le même objet. La circulation libérée du désir n'induit pas nécessairement une circulation homogène des flux qui peuvent se concentrer, converger sur une même personne au lieu de faire correspondre chacun avec chacune. Le désir est par nature complexe, même s'il se donne sous l'apparence d'une relation simple de l'un à l'autre, comme un rapport (réciproque) de sujet à objet. La relation est rarement simple relation duelle, elle n'est telle que de façon exceptionnelle. Bien souvent, et même si au départ il s'agit effectivement d'une relation duelle, il y a la présence d'un autre, ou des autres, spectateurs et bientôt acteurs. Les autres deviennent des tiers ; le regard n'est jamais la pure extériorité du regard, il est aussi désir de participation, désir d'être à la place de l'un ou de l'autre, désir d'intromission dans une dualité trop close pour demeurer telle trop longtemps : «Cecca - Madame Gostanza, cet éloge... vous faites honneur à Panfilo Teodosi. ${ }^{7}$ Les deux commères sont dans un rapport de surveillance réciproque. Le contrôle social s'exerce en l'absence du mari, qui est d'ailleurs mentionné comme garant du bon comportement: Gostanza sous-entend que son honnêteté d'épouse est une évidence sur laquelle il n'y a même pas à discuter. Cet état d'épouse, et la conscience des obligations qui lui sont liées, garantirait un nondésir : l'intérêt marqué pour les deux jeunes hommes masqués - mais identifiés ${ }^{8}-$ ne cache rien d'autre qu'un intérêt « générique », ne s'adressant d'ailleurs à aucun des deux en particulier. C'est d'ailleurs cette absence de particularisation du désir qui assurerait qu'il s'agit d'un désir dénué de connotations sexuelles, un intérêt « humain » en quelque sorte, sans aucun investissement d'objet. Le rappel fait par Gostanza de la situation de femme mariée est conscience permanente de son état et de son statut social, ainsi que du rôle qui lui incombe : mais c'est en même temps un rejet du regard trop inquisiteur de $\mathrm{Cecca}^{9}$, une façon de dire, à la première personne, la haute conscience du devoir qui est la sienne. Cecca n'exerce pas son contrôle pour le simple plaisir de la maîtrise qu'il peut conférer sur autrui. Elle est, sans nul doute, mue par des motivations de la même nature que celles qu'elle dénonce chez sa voisine. C'est cette identité de motion qui en déclenche la lecture aussi prompte et aussi perspicace chez l'autre. Enoncer la réalité du désir, c'est pour Cecca la dénoncer. Mais c'est également parler pour les autres : Cecca puise

\footnotetext{
${ }^{7}$ VA, p. 58.

${ }^{8}$ Cecca a reconnu au moins Panfilo Teodosi (étrange paronymie entre Teodosi qui est le patronyme d'Arrigo, père de Panfilo et ami ainsi que complice de Teodoro. Il y a entre eux comme une relation de doubles, si ce n'est une quasi-identité, dont nous verrons qu'elle renforce le caractère incestueux du désir de Teodoro pour la fiancée de Lionetto).

9 «-Cette façon que vous avez de tant les louer me laisse croire que vous les connaissez et que vous y mettez de la passion. »VA, p. 58.
} 
dans la collectivité la force de son discours. Ce qu'elle dit est vrai parce que d'autres le pensent également ou l'ont constaté. Mais dénoncer a surtout pour but d'empêcher. Cecca barre le désir de Gostanza en s'autorisant de la collectivité (celle du voisinage, qui peut voir). Mais cela se révèle insuffisant, et cela intervient dans l'échange qui se noue, contre son gré, entre Panfilo et Gostanza :

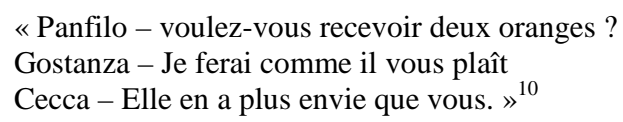

Le déséquilibre souligné par Cecca a un objectif de dissuasion; Cecca intervient juste après une phrase de Gostanza qui est, il est vrai, riche d'ambiguïté : il y a ponctuellement l'acceptation de l'offre de Panfilo, et c'est là une réponse de courtoisie à un acte rituel, s'insérant dans le rituel plus vaste du carnaval tel qu'il est pratiqué à Pise. Il y a aussi une acceptation bien plus générale, une sorte de mise à disposition d'autrui opérée par Gostanza. Le désir de se conformer au désir de l'autre témoigne de la totale disponibilité de Gostanza, de son accueil sans réserve. Mais probablement avons-nous là également un effet de la volonté de censure de Cecca : c'est parce que celle-ci a dénoncé le lien spontané pour empêcher la réalisation du désir que Gostanza déclare aussi ouvertement et de façon aussi démonstrative sa disponibilité à l'égard de Panfilo. La tentative d'inhibition, menée par Cecca, a en vérité fourni l'occasion d'une formulation du désir qui s'adresse autant à Panfilo qu'à Cecca. Gostanza fait ainsi d'une pierre deux coups : elle peut dire son désir à celui qui est concerné, et elle peut infliger un camouflet à celle qui a manifesté sa jalousie et qui se trouve du coup confinée dans une position purement défensive, sans autre effet que celui de la dénonciation de ce qui vient déjà de s'énoncer. La surdétermination du désir par la parole - au lieu de sa litote - ne fait que redoubler l'affirmation qui a déjà eu lieu, et elle renvoie Cecca à la position qu'elle a adoptée initialement, celle d'observatrice critique, par là même rejetée à l'extérieur de la relation duelle. S'il est vrai que c'est la rivalité qui a suscité le comportement de contrôle social, Cecca s'est mise par ce choix en dehors du cercle de la compétition; qui plus est, elle a attisé la manifestation du désir de Gostanza et elle s'est trouvée refoulée à la marge de la relation qui se noue entre Panfilo et Gostanza. Elle n'a pas pu ou pas su utiliser le contrôle social à des fins personnelles ; elle n'a pas pu écarter Gostanza, bien au contraire. Et elle qui a voulu rappeler à l'ordre, et condamner, se retrouve hors jeu. Mais ceci ne signifie pas que le contrôle social ne s'exerce pas : simplement, il ne passe pas par Cecca, mais par la belle-mère qui fait son retour. L'efficacité de sa présence n'est pas due à une autorité personnelle. En tant qu'individu, la bellemère n'aurait probablement pas plus de pouvoir de contrôle social sur Gostanza que Cecca, puisque Gostanza a affirmé détenir en elle-même les éléments qui permettent de savoir où se situent les limites de son comportement, puisqu'elle s'affiche comme une femme ayant conscience d'elle-même, c'est-à-dire une femme liée à un mari, avec toutes les obligations et tous les interdits que cela comporte. L'efficacité de la belle-mère tient à sa position de belle-mère, en tant

${ }^{10}$ VA, p. 58. 
que victime possible, donc en tant que juge des conséquences des comportements de sa belle-fille. Mais si l'on approfondit la situation, on découvre que la position de belle-mère confère une partie de l'autorité maritale : la mère devient alors, mais momentanément seulement, la représentante de son fils (le mari). L'autorité réelle de la femme qu'elle est n'est qu'une autorité dérivée ; c'est l'homme qui est le support véritable de toute autorité, c'est lui qui au bout du compte évalue toute transgression, c'est lui qui, implicitement ou explicitement, fixe à chacun les limites possibles de son autonomie. Face au contrôle social, chacun détient une marge d'autonomie, mais non point une liberté dont il serait en mesure de fixer ou de déplacer à son gré les bornes. L'autorité, articulée sur la fonction maritale et la fonction paternelle est le noyau du contrôle social, c'en est le point de départ et le point d'arrivée : tout part de ce point central du système social, et tout y revient, de façon convergente, pour confirmer sa place et renforcer son pouvoir.

\section{Le contrôle social comme discours}

De quelles médiations s'entretient l'autorité, de quels moyens dispose-t-elle pour être autorité en acte, c'est-à-dire contrôle effectif? Telles sont les interrogations auxquelles il convient, à ce point de nos observations, de répondre.

L'autorité ne requiert pas toujours une médiation. Par exemple, elle s'exerce directement du mari sur sa femme :

«Teodoro - Comment, je veux savoir trop de choses! Les femmes peuvent-elles faire quelque chose sans leurs maris ne s'en aperçoivent.

Dianora - Non, mais... ! Vois ce qu'il me dit ! Comme si on ne savait pas comment je me conduis. ${ }^{11}$

L'autorité n'a de sens que porteuse d'effets. Pour cela elle a besoin du contrôle, elle a donc besoin de savoir. C'est ce que réaffirme Teodoro. Son droit de savoir découle (pour lui) naturellement de sa position d'homme et de mari, préposé à la surveillance des actes de sa femme, qui sont susceptibles de porter du tort à sa réputation. Cette exigence de transparence fait peser le soupçon de la faute sur tout ce qui n'entre pas directement dans le champ du savoir ; toute ombre est probabilité ou risque de culpabilité. C'est l'extension de la suspicion qui voudrait contraindre l'épouse à tout dire, si ce n'est à tout avouer ! Là où le mari est, de son propre point de vue, fondé à craindre des comportements infamants pour lui, il menace son épouse de reverser sur elle l'infamie qui en réalité n'est pas. Teodoro n'a pas face à lui quelqu'un qui se trouverait en mauvaise posture. Et on voit bien le renversement psychologique qui a été opéré : c'est celui qui s'apprête à fauter qui émet à propos de l'autre le soupçon de la faute. Dianora peut s'appuyer sur des certitudes, et continuer à faire ce qu'elle veut garder pour elle, sans encourir le risque d'aucune mise en accusation. La réplique de Dianora annihile sur ce point (crucial) l'autorité dont son mari a voulu se prévaloir. Si on examine attentivement la nature des certitudes sur lesquelles s'appuie Dianora, on trouve certes une certitude intérieure faite de la conformité des actes aux impératifs de la conscience morale, faite également d'une paix intérieure qui n'a

\footnotetext{
${ }^{11}$ VA, p. 29.
} 
besoin d'aucune argutie pour démontrer son bien-fondé, et qui, par là, peut se contenter de s'affirmer elle-même. Mais il y a plus : cette certitude n'est pas solipsiste, elle trouve une confirmation extérieure constante, constituée en un véritable savoir sur Dianora : le sujet de cette certitude extérieure, qui apporte un appui objectif à la certitude intérieure, est un sujet collectif : « on », le « on » de l'expression de Dianora lorsqu'elle dit «comme si on ne savait pas comment je me conduis ». A la suspicion du mari, elle a opposé la voix des autres, la voix d'une collectivité anonyme garante de ses actes, témoin omniprésent capable de voir, en tout moment et en tout lieu, capable d'interpréter le moindre indice - ou plutôt de transformer tout signe en indice -, et également capable de dire et de donner acte de ce qui ne s'est jamais vu. Là réside la vraie certitude de Dianora, celle que quiconque pourrait énoncer contre les insinuations de Teodoro. L'autorité à laquelle se réfère Teodoro, sur laquelle il fonde son droit à l'investigation, n'est rien sans cette médiation du discours des autres. L'autorité seule est sans relais si elle n'est pas en accord avec le « discours courant », avec ce qui se dit et tient lieu de vérité. Dianora est en règle et en paix avec le contrôle social ; les velléités tatillonnes de son époux ne valent rien contre la force d'une telle certitude.

Le contrôle social a donc un relais : l'opinion des autres. Chacun est, dans l'abstrait, libre de ses actes, dans le sens où une volonté bien formée ne saurait rencontrer aucun obstacle qui la détourne réellement du but qu'elle s'est donné. Mais la liberté va de pair avec la responsabilité. Tout acte a un prix : ceci vaut d'une façon générale. Mais tout acte « répréhensible », c'est-à-dire contraire aux règles morales en vigueur, a un coût élevé, qu'il appartient à l'individu responsable de payer, souvent de façon irréversible et à vie, car certains actes ouvrent envers la collectivité une dette impossible à solder. La «faute » laisse une trace, indélébile parce qu'elle n'est rien d'autre qu'une inscription dans l'esprit et surtout dans la mémoire des autres. Et les plus exposées à la «faute » sont les femmes, lorsque la faute se définit principalement dans le domaine de la morale sexuelle. Ainsi, lorsque Lionetto raconte à son ami que l'esclave Diamante qu'il ramène de Palerme est enceinte de lui, Panfilo fait la remarque suivante :

«- car en vérité, c'est une grande cruauté que d'enlever son honneur à une pauvre jeune fille, de quelque condition qu'elle soit, et ensuite de l'abandonner. ${ }^{12}$

Un déséquilibre flagrant apparaît ici entre hommes et femmes. La responsabilité de la naissance d'un enfant hors mariage ne retombe que sur l'un des deux responsables réels. Symboliquement, la société ne connaît - et ne reconnaît, ici négativement - qu'un coupable, la femme. Une femme «sans honneur » ne peut plus s'insérer dans l'échange social, celui qui a lieu à travers le mariage ; car l'échange affectif entre les individus ne vaut rien s'il n'est pas inscrit dans le code social et institutionnel des échanges. La femme qui a perdu son honneur (l'honneur dont on la tient pour la gardienne et la responsable) sera une exclue, vivante, mais morte à l'échange social, morte à la vie de famille, seul lieu d'épanouissement possible pour une femme pendant de nombreux siècles de notre

${ }^{12}$ VA, p. 4. 
civilisation. Et qu'est l'honneur, si ce n'est le discours que les autres tiennent sur un sujet, discours avec ses connotations et ses effets de mise à l'écart symbolique, et, par voie de conséquence, réels ? Dans le cas de figure qui nous intéresse, l'homme semble être à l'abri du discours qui déshonore ${ }^{13}$. Seule la femme y est assujettie parce que son éventuelle dénonciation serait sans effet : non seulement pour des raisons matérielles de preuves, mais aussi pour des raisons symboliques : son discours est disqualifié par avance et il n'a donc pas lieu d'être. En cela consiste la cruauté qu'évoque Panfilo, celle d'une impunité assurée pour celui-là même qui ne laisse derrière lui que le déshonneur qui s'abat sur celle qui s'est donnée à lui, le plus souvent par amour. On reconnaît ici la vieille accusation biblique de perversion féminine : tout ce qui a lieu hors des sacrements du mariage ne peut être que péché de chair, né de l'abandon à la concupiscence.

La réprobation qui touche la femme coupable est susceptible d'extension :

« Teodoro - Outre cela, est-ce qu'il te semble convenable que je garde une jeune fille d'une telle beauté, alors que tu n'as pas encore pris femme?

Lionetto - Jusqu'à présent, je n'ai jamais rien fait qui ait causé la moindre honte à notre maison. $»^{14}$

Teodoro joue ici son rôle de garant de l'honneur de la maison. Il refuse la venue de Diamante: sa trop grande beauté serait source de soupçons. Teodoro serait alors suspecté de prêter la main à des actes que la morale réprouve. Chacun est alors comptable de l'honneur de toue la famille ${ }^{15}$. Mais l'essentiel de la contamination consisterait en l'extension à tous du déshonneur lié aux actes coupables supposés (et imputés) entre Lionetto et Diamante. On ne manquera pas d'observer que ce qui est déshonneur pour la femme peut être honte pour la famille. Or la honte, la difficulté, si ce n'est l'impossibilité d'affronter le regard des autres. La honte, c'est ce qui ne peut être assumé face aux autres. La honte se profile donc comme l'horizon négatif de la responsabilité individuelle et familiale. L'exercice du contrôle social consistera en l'évitement de tout acte susceptible de conduire à une telle extrémité, il sera préservation du «capital », du «crédit» de bonne opinion et d'estime ${ }^{16}$ dont la famille est bénéficiaire ${ }^{17}$. C'est sur cette exigence que Teodoro joue, peut-être même sans le savoir, dans le conflit d'intérêts et dans la course à l'objet qui l'oppose à son fils Lionetto ; quoi qu'il en

\footnotetext{
${ }^{13}$ Au moins pour ce qui concerne la visibilité (la naissance d'un enfant) de la vie sexuelle. Ce qui peut déshonorer l'homme, ce sont les comportements indus d'un membre de sa famille, en particulier de sexe féminin. L'opprobre qui s'abat très facilement sur une femme s'étend, comme métonymiquement à tous ceux qui sont sous le même patronyme qu'elle. Mais le déshonneur sui generis d'un homme consiste à ne pas savoir défendre face à d'autres hommes, y compris par un combat s'il le faut, tout affront porté à son image publique. La forme la plus exacerbée de cet honneur réside dans le «point d'honneur» (puntiglio) qui guide toute la conduite publique des nobles.

${ }^{14}$ VA, p. 32.

${ }^{15}$ Le déshonneur a une réelle puissance de diffusion, d'extension à tous les sujets porteurs d'un même patronyme (cf. note 13).

${ }^{16}$ L'estime et l'opinion, sous la forme de capital de départ sont bien entendu un «préjugé » au sens où René Girard promeut cette notion.

${ }^{17}$ C'est ainsi que se définit la «prudence » : « Teodoro - Les hommes prudents doivent toujours éviter les occasions de faire le mal [...] Je ne la veux pas à la maison. »VA, p. 32.
} 
soit, que Teodoro en ait conscience ou non, il n'en demeure pas moins que les choses apparaissent à Lionetto sous la forme d'une contradiction ou même d'une impossibilité :

«- Et si je veux m'opposer à lui, je le ferai passer pour fou : ce qui ne sera pas sans déshonneur pour moi. Car au bout du compte, la honte des pères retombe sur leurs fils. ${ }^{18}$

La famille constitue une continuité, une unité dans les signes. Les actes répréhensibles des enfants rejaillissent sur tous les membres de la famille selon un principe de coresponsabilité ; comme Teodoro en tire argument pour ne pas vouloir Diamante chez lui (et ainsi la soustraire à son fils), la mauvaise réputation des parents rejaillit naturellement sur les enfants. Si Lionetto veut récupérer Diamante, la seule façon de combattre l'autorité institutionnelle de son père, c'est de la lui faire retirer de façon tout aussi institutionnelle : il s'agirait alors de faire reconnaître l'irresponsabilité (mentale) de Teodoro, pour que celui-ci soit du même coup déchu de sa responsabilité (sociale) de père de famille. Lionetto aurait alors la responsabilité de l'irresponsabilité indirecte dans laquelle il serait tenu par les autres. Il y a une contamination naturelle, qui court le long de la chaîne des signifiants, et qui s'étend du père au fils, selon une logique sociale, tacite mais effective, comme elle peut aussi circuler du fils en direction du père. La famille est, sur le plan réel et matériel, une chaîne de solidarité, comme elle l'est aussi sur le plan symbolique. On n'est pas impunément, dans l'esprit des autres et dans la représentation qui s'attache au patronyme, le fils d'un homme fou. Lionetto ne peut disqualifier l'autorité de son père sans, par là même, se disqualifier lui-même.

Cette aporie paralyse son action, elle le contraint à trouver un autre cheminement qui ne passe pas par la médiation symbolique des autres. Et comme toute démarche à caractère symbolique impliquerait nécessairement les autres, il ne restera à Lionetto que la voie d'une action réelle et directe, qu'il entreprendrait seul ou avec un nombre restreint de complices.

Si Lionetto ne peut recourir au regard et au discours des autres contre son père, il est, pour la réalisation de son projet, dans l'obligation de se dérober au regard des autres; c'est ce que précise Panfilo : «Et de pareilles choses, il est bien qu'elles se fassent de façon occulte, en raison du blâme qu'elles entraînent. ${ }^{19} \mathrm{On}$ voit l'adhésion de Panfilo au projet de Lionetto pour des raisons amicales d'une part, et d'autre part pour des raisons de reconnaissance de la légitimité de ses intentions. Panfilo établit la distinction entre les actes et le jugement sur les actes. La complicité de Panfilo dans l'hébergement de Diamante, et par voie de conséquence, dans les rencontres qui s'ensuivront entre Lionetto et Diamante lui paraît en soi une chose juste, au service d'une cause tout aussi juste, celle de l'amour de deux jeunes gens honnêtes dans leurs intentions l'un envers l'autre. Le blâme qui peut en résulter si la chose se savait, la condamnation par la société est enregistrée comme une donnée objective de la situation avec laquelle il faut compter. La démarche de prudence, la volonté que les choses soient, mais sans être vues ni sues, attestent la non-adhésion aux valeurs qui circulent, au nom

\footnotetext{
${ }^{18}$ VA, p. 20.

${ }^{19}$ VA, p. 16.
} 
desquelles les libres relations entre les jeunes doivent recevoir, pour pouvoir être, l'aval des parents et des plus vieux en général. L'action est perçue comme une source de blâme, pragmatiquement - elle n'est pas pensée comme méritant, en soi, le blâme. Sur l'autre versant de l'action, on trouve le même comportement : pour échapper au contrôle social, le complice (on note le parallèle dans la situation) suggère la prudence : «Arrigo - Ne fais pas cela ; parce que si nous étions vus, on dirait que nous sommes devenus gâteux. ${ }^{20}$ Ceux-là mêmes qui sont situés au point nodal de la circulation et de l'exercice de l'autorité sont obligés de compter avec le contrôle social. Et le risque pour eux est d'autant plus grand que leur position exige l'exemplarité des comportements. Ce qui peut s'offrir au regard des autres comme modèle extensible à tous, doit donc ne pas être (exemplarité négative, par abstention) ou ne pas paraître (pseudo-exemplarité négative). Si le désir d'atteindre l'objet convoité n'est pas d'une force égale à l'exigence (ou à la contrainte) de moralité, si les impératifs laissent un résidu tel que le désir subsiste en partie ou en totalité, le sujet va opter pour un compromis de pure forme entre ses propres attentes et celles de son groupe social, et il va résorber la tension de ces forces antagonistes en produisant les apparences d'une réalité conforme à ce précédemment nous avons désigné comme l'exemplarité négative ou par abstention; car le risque pour les deux compères âgés, c'est la disqualification, celle qui dans certains cas frappe les gens de leur âge que leurs capacités mentales amoindries ne placent plus à la hauteur du rôle (et du pouvoir) qui peut et doit être le leur. Teodoro et son complice ont donc à craindre, plus que toute autre chose, le regard des autres et le dire qui s'ensuivrait. Et pour que le dire n'advienne pas, alors que l'agir coupable est maintenu comme projet, il convient d'éviter le regard. Qu'une chose ne fasse pas signe, et elle pourra être, presque librement, tout en échappant au contrôle exercé par la collectivité.

Le manquement aux règles sociales s'observe chez d'autres détenteurs de l'autorité, les gens d'Église. Le représentant de ces acteurs sociaux à l'intérieur de la pièce se plaint de la différence qui sépare religieux et laïcs :

«- Ces laïcs se donnent bien plus de bon temps que nous ne le faisons, nous autres religieux, car nous sommes soumis non seulement à nos règles mais aussi aux regards de chacun, et nous manquons de bien des aises [...] Si on nous voyait profiter de un seul de ces plaisirs ils nous lacéreraient jusqu'aux os, sans prendre en considération le fait que notre chair et nos nerfs nous titillent comme les autres. Si bien que cela fait belle lurette que, si j'avais ou le faire honorablement, j'aurais jeté ces habits aux orties ! Oh, je suis toujours bien vert, malgré ceux qui veulent le contraire et malgré les mauvaises langues; et ces habits me sont d'une aide précieuse pour avaler de bonnes bouchées. Il faut certes agir avec circonspection : et pour cela, je ne manque pas d'esprit. $»^{21}$

Les laïcs auxquels le prieur se réfère sont les deux jeunes hommes, Lionetto et Panfilo, auxquels il a prêté refuge pour qu'ils puissent se déguiser, à l'occasion du carnaval et du rite des oranges. Une nouvelle fois, le carnaval permet une subversion des valeurs, ici involontaire: c'est au sein même de l'Église que s'organise la réjouissance profane, c'est près du lieu de la pureté et de la nudité de

\footnotetext{
${ }^{20}$ VA, p. 25.

${ }^{21}$ VA, p. 55. La lapidation est l'une des punitions infligées à la victime émissaire.
} 
l'âme face à Dieu que s'organise la mascarade par laquelle chacun va apparaître comme autre que celui qu'il est. Et la subversion des valeurs, involontaire peutêtre, mais bien réelle, tient à l'effet de miroir s'établissant entre le déguisement des deux jeunes et le vêtement du religieux, qui souffre d'un déficit de considération (mais aussi d'un déficit d'adhésion à sa fonction sacerdotale). Le prieur se plaint d'être soumis, à la différence des autres, à un double contrôle social : un contrôle interne (la discipline religieuse, librement consentie) et un contrôle externe (le regard de tous les autres, laïcs). Ce faisant, il ne se rend pas compte d'une chose : c'est le contrôle interne qui fonde l'autorité morale des religieux, et c'est lui aussi qui fait leur position particulière dans les signes, sous le regard. Il est vrai que le vêtement est la marque la plus évidente, la plus distinctive de cette vocation au contrôle social. Le vêtement désigne, en vérité, celui que marque l'exemplarité sociale, celui qui devrait être le signe et le rappel d'une autre Présence, exemplaire par-delà toutes les autres. Le manquement à l'exemplarité est fortement sanctionné, le discrédit en est la conséquence et la sanction. Ceux qui sont, institutionnellement, en position de maîtrise, sont exposés, et naturellement plus exposés, en particulier au risque de victimisation émissaire :

«- Si nous faisons bien, on ne nous croit pas; si nous faisons mal, nous sommes mis en croix. Si en cette période de carnaval on nous voyait nous amuser, je crois que les enfants nous poursuivraient en nous lançant des pierres. $»^{22}$

Les religieux, en tant que catégorie sociale, semblent souffrir du plus grand discrédit: le bien comme le mal reçoivent une pareille interprétation, tout au moins le bien ne fait pas l'objet d'un crédit en faveur des religieux. Tout bien qui apparaît est rejeté dans la pure apparence du bien : la circulation et la stricte équivalence de l'être et du paraître n'ont plus cours. Le mal fait l'objet de la plus grande rigueur: c'est que la société pardonne mal aux ministres du contrôle idéologique de la population de ne pas se soumettre aux mêmes règles que celles auxquelles ils assujettissent les autres ${ }^{23}$. La source du contrôle est alors inversée, et, de ce fait, renforcée : elle s'exerce en provenance de la population à l'encontre des ministres du culte. Et la rigueur de la condamnation s'explique par le déficit d'exemple dont ils sont la cause. C'est le manque à imiter, c'est ce défaut d'idéalité que les autres sanctionnent si vigoureusement chez les religieux. Par ses paroles, le prieur révèle en réalité une situation de crise, et ce à double titre : tout d'abord parce que se manifeste une fracture entre le sujet et son rôle social; le sujet n'est pas totalement identifié à son paraître, il n'assure pas, dans sa plénitude, la fonction de contrôle à l'égard des autres et qui devrait être en lui une idéalité vivante, une aspiration de tous les instants et un exemple infaillible. Ensuite, la direction de l'exemplarité est subvertie: le prieur implicitement revendique le droit au rituel païen du carnaval, que les autres lui interdiraient sous peine de vive condamnation. C'est bien se situer en rivalité avec les autres, convoiter le même objet licencieux qu'eux, et être pris du même esprit profane que les laïcs eux-

\footnotetext{
${ }^{22}$ Ibid.

${ }^{23}$ C'est en même temps le coût individuel pour tout sujet participant au contrôle social : il paie de sa propre abstinence, de son rigoureux respect des règles, la gratification d'être celui par qui le respect des règles est rappelé ou éventuellement sanctionné.
} 
mêmes ; c'est placer les autres en position de modèle et - heureusement - de modèle-obstacle. La collaboration du prieur au travestissement des deux jeunes hommes est, à ce titre, significative : elle est une participation vicariante au rituel profane, elle est un agir par défaut et surtout par empêchement. L'autorité est réellement en crise : le contrôle est exercé à rebours par ceux-là mêmes qui devraient être sous contrôle, il provient d'une instance extérieure au lieu de s'exercer spontanément et positivement comme instance intérieure: non pas comme instance surmoïque mais comme idéal du moi. Nous sommes bien loin d'une autorité en harmonie avec elle-même, qui exercerait, par simple rappel verbal, sa fonction de contrôle social. L'autorité est déchirée, tiraillée intérieurement $^{24}$, et elle se déploie dans le réel de façon contradictoire : le prieur seconde les jeunes dans le projet qui les unit contre un autre pilier de l'autorité, les pères.

\section{Le dévoiement du contrôle social}

Tout autant que le prieur se sert de l'habit sacerdotal pour en tirer des avantages personnels ${ }^{25}$, les pères revêtent les habits de l'autorité paternelle au service de desseins tout à fait personnels, et sans rapport avec cette autorité ellemême et son exercice au profit de la collectivité et de son équilibre. Il existe une hostilité de fond, une indépassable rivalité qui oppose les pères aux fils ; il semblerait que les pères, Teodoro en particulier, soient jaloux du bien-être économique qu'ils assurent à leurs fils. La contrepartie qu'ils exigent, pour compenser les avantages qu'ils procurent à leurs enfants, c'est une obéissance qui prend la forme d'une acceptation soumise des caprices qui privent les jeunes des plaisirs auxquels ils peuvent légitimement aspirer :

«- Que les jeunes s'occupent activement; et lorsqu'ils se seront échinés un bon moment et qu'ils auront acquis du bien, qu'ils se donnent alors aux plaisirs ; comme je l'ai fait, moi et tous ceux qui possèdent quelque chose. $\gg^{26}$

Les jeunes doivent donc mériter leur autonomie, et avec elle le droit au plaisir. La raison économique justifie tous les abus de pouvoir, et le maintien de tous les interdits ; mais surtout la raison économique sert à fonder, dans le champ du réel, la pulsion d'emprise, étendue par Teodoro à l'objet sexuel que Lionetto convoitait. L'attirance ressentie par Teodoro à l'égard de Diamante vaut sur les deux versants de la relation triangulaire : obstacle posé à Lionetto d'une part, et maîtrise assurée sur l'objet sans aucune considération d'ailleurs de la réciprocité de l'attirance ${ }^{27}$. L'autorité des vieux devient de la sorte gérontocratie, où tout est prétexte à la réaffirmation du pouvoir, pâle compensation à toutes les frustrations vécues durant

\footnotetext{
${ }^{24}$ Cf. cit. note 19 : «notre chair et nos nerfs nous titillent comme les autres ».

${ }^{25} \mathrm{Cf}$. « ces habits me sont d'une aide précieuse pour avaler de bonnes bouchées », ibidem.

${ }^{26} \mathrm{VA}$, p. 26.

${ }^{27}$ Moro, le valet de Teodoro, rapporte à Lionetto comment son vieux maître a voulu embrasser Diamante, sans se demander si elle le souhaitait elle-même : "-Après qu'il eut parlé un certain temps avec elle, il s'apprêta, sans tenir compte de ma présence, à l'embrasser. Mais elle fut prompte, et elle protégea son visage de ses mains, et elle s'éloigna de lui », VA, p. 19.
} 
la jeunesse et transmises ainsi à la génération suivante ${ }^{28}$. «Tout a un prix », tel est le discours de Teodoro, et c'est lui qui se pose en personne déléguée à la perception de cette dette. La rivalité entre vieux et jeunes est également symbolique :

«Lionetto - ... ne sais-tu pas que par nature, les vieux s'opposent toujours aux volontés et aux décisions des jeunes, ne serait-ce que pour paraître plus sages ? ${ }^{29}$

La rivalité s'est déplacée du plan de l'être à celui du paraître. La maîtrise symbolique est à ce prix pour eux. L'obstacle qu'ils érigent entre les jeunes et la réalisation de leurs projets n'est par véritablement fondé en justice, pourrions-nous dire. La notion d'interdit apparaît ainsi très fluctuante, variable au gré du caprice des vieux. L'interdit est toujours défini a posteriori, parce que personne n'est en mesure d'en formuler les contours a priori, pour la bonne raison que peu d'arguments - si ce n'est aucun argument - peuvent valablement étayer l'interdit. La dénégation que les pères opposent à tout projet reste profondément arbitraire, et elle cache un renversement: alors que normalement c'est le mal que définit l'interdit, il advient que c'est l'interdit qui désigne le mal. Alors le mal n'est rien d'autre que l'objet désiré, quel que soit cet objet ${ }^{30}$, et il est frappé d'interdit parce qu'il est investi par le désir d'un autre (le fils). La supériorité symbolique que les pères s'attribuent en se faisant passer pour plus âgés que leurs fils renforce à rebours, en apparence, si ce n'est en réalité, leur pouvoir d'interdire. Une fois cette circulation dénoncée, l'autorité des vieux est discréditée : elle ne se présente plus que comme un obstacle à surmonter, obstacle doublé d'une pseudo-justification symbolique et de menaces réelles concernant la disposition des biens (héritage), avec la menace de castration symbolique dont une telle privation entend inconsciemment être porteuse. Il est vrai que Lionetto n'a pas attendu ce dernier avatar de sa relation avec son père pour comprendre à quoi l'autorité dont Teodoro est investi sert de masque. La première lueur de la prise de conscience est probablement apparue dès le moment où Teodoro l'avait éloigné de Pise, à cause de la relation qu'il entretenait avec Giulia, une jeune fille de Bologne : c'est ce que laisse comprendre l'analyse qu'il fait de la situation, peu de temps après son retour, avant que Teodoro ait mis en place son stratagème :

« - .. avec l'aide de ma mère. Entre-temps, soit il mourra (parce que maintenant il est vieux), de sorte que je pourrai l'épouser et faire ce que je veux; soit il se passera quelque chose qui me donnera l'occasion de me satisfaire et de le satisfaire. $»^{31}$

Le spectateur pourrait s'étonner de la froideur avec laquelle Lionetto envisage la mort de son père : celle-ci est évoquée comme un événement du monde, neutre, non porteur d'affects chez le personnage. Le père a pour toute épaisseur psychique la matérialité de l'obstacle, et rien de plus. Lionetto semble émancipé de l'emprise de toute imago paternelle, et, de toute façon, le père symbolique et le père réel ne

\footnotetext{
28 « Arrigo - Mais pourquoi les [ces choses malhonnêtes] fais-tu maintenant que tu es vieux ?

Teodoro - Parce que je ne les ai pas faites quand je devais les faire. »VA, p. 38.

${ }^{29}$ VA, p. 15.

${ }^{30}$ Derrière l'objet, la rivalité porte sur la jouissance.

${ }^{31}$ VA, p. 15.
} 
coïncident pas pour lui en la personne de Teodoro. Toute l'affection filiale de Lionetto semble avoir pour support et pour objet sa mère, Dionora. Il est assuré par avance de sa participation au projet qu'il lui soumettra. Une complicité semble les unir, indépendamment de Teodoro, ou même contre lui, et surtout contre les mauvaises résolutions qu'il est capable de prendre à leur égard. Teodoro n'est ainsi qu'un «empêcheur », le représentant concret d'une autorité vécue comme lointaine, et dans certains cas immotivée. Teodoro est plus que tout autre chose un pouvoir, qui revêt l'apparence de la dignité de l'autorité sociale mais il ne fait que dévoyer, à son propre usage et à son propre profit, la délégation de contrôle qui lui est dévolue par la société. C'est parce que le jeu de Teodoro est mis à jour, parce que les masques sont tombés sans la moindre ambiguité, que ce père peut faire l'objet d'une action concertée des autres. Le père réel ne jouit désormais plus de la protection du père symbolique : Teodoro a perdu toute épaisseur morale aux yeux de son fils et de sa femme, il est simplement un individu, à la limite même un étranger, agissant à partir d'une place privilégiée (mais moralement usurpée) qui lui confère une possibilité de nuire à autrui, et en particulier à son entourage. Son comportement à l'égard de Diamante, le projet de séquestration à son endroit, met en jeu les «intérêts », psychologiques essentiellement, de sa femme Dianora et de son fils. Logiquement, les actions de ces deux victimes des agissements de Teodoro vont converger et s'étayer réciproquement. Elles deviendront contrôle social de fait; il en résultera une désignation de Teodoro au regard des autres, qui pourrait bien signifier sa mort sociale. C'est à cette désignation que doivent se résigner Lionetto et son complice Panfilo: «- Toute la honte est pour nos vieillards, envers lesquels on n'a plus à avoir aucun respect. ${ }^{32}$ La précision apportée sur le respect qui ne s'impose plus comme impératif moral a toute son importance, parce qu'elle marque le point de départ des réflexions de deux jeunes gens en même temps que la rupture - «épistémologique » pourrions-nous dire par métaphore - qu'ils opèrent. Lionetto et Panfilo n'ont plus à se soucier de la mauvaise réputation qui leur reviendrait indirectement, s'ils jetaient le discrédit sur leurs pères. Ils ont décidé d'écarter la procédure du recours au contrôle des autres. Lionetto va pouvoir se faire justice lui-même, il va pouvoir récupérer par ses propres moyens son bien (Diamante) en mettant à profit le désordre que permet la période de carnaval, et surtout en utilisant l'anonymat que permet le travestissement. Panfilo et Lionetto n'ont plus à avoir de crainte de subir l'opprobre qu'ils n'ont en aucune manière méritée. Le contrôle social a montré ici son efficacité, puisqu'il a conditionné le choix de la stratégie adoptée par les deux jeunes hommes, en leur faisant préférer le moyen qui ne causerait aucune sanction en termes de discours et d'opinion des autres.

Mais avant même que Lionetto s'affaire à récupérer, de fait, son bien, sa mère s'efforce d'obtenir l'assentiment du père :

«Dianora - Si vous ne donnez pas satisfaction à ce pauvre petit, j'allumerai un tel feu entre vous et toute ma famille qu'il brûlera jusqu'au ciel. Et alors vous verrez qui est Dianora. Et je veux que vous sachiez qu'elle est enceinte de lui; et je n'entends pas que l'on fasse commerce

\footnotetext{
${ }^{32} \mathrm{VA}$, p. 62.
} 
de notre sang. Vous croyez m'avoir toujours à votre botte. Cela ne marchera pas. Moi, je suis une Lanfranchi. ${ }^{33}$

La force de Dianora, qui compense l'autorité et le pouvoir que Teodoro détient envers elle, consiste dans la possibilité du recours à des tiers, qui sont les membres de sa famille d'appartenance biologique: Teodoro reste comptable devant eux du pouvoir qui est le sien, tout abus portant déshonneur à Dianora étant passible d'une riposte de leur part. C'est l'éventualité d'un conflit généralisé, c'est-à-dire de désordre maximal (hyperboliquement de dimensions cosmiques) que Dianora laisse entrevoir. Teodoro se trouve confronté à une situation tout à fait négative pour lui : d'une part le scandale qui ne manquerait d'éclater grâce aux bons soins de la famille de Dianora s'il persistait dans son infâme projet, d'autre part une relation sans issue possible, puisque Diamante est enceinte d'un autre (son propre fils) $)^{34}$, avec, pour Teodoro, un nouveau sujet de scandale possible, à savoir la naissance d'un enfant que tout laisserait croire sien. On imagine alors l'ampleur du remue-ménage que la famille pourrait, sur la base de cet indéniable outrage, provoquer à l'encontre de Teodoro, et l'assentiment général que recueillerait Dianora contre lui. L'autorité dont Teodoro est investi n'aurait plus aucune efficacité, et le discrédit qui en découlerait pour lui serait directement proportionnel à la «visibilité » de sa position sociale. Cependant, au moment où ce dialogue se tient, Teodoro ignore que Dianora sait parfaitement les intentions qui l'animent. Il ne perçoit donc pas, ou il ne perçoit pas comme s'adressant à son projet secret, la réalité des menaces que lui adresse son épouse. Il ne voit pas que Dianora, en protégeant son fils, se protège elle-même, puisque l'action entreprise par le père nuit autant au fils (mal psychologique et réel) qu'à la mère (psychologique et mal symbolique: elle devient femme bafouée). Si Dianora obtient de Teodoro qu'il consente au mariage de son fils avec Diamante, puisque au bout du compte c'est bien de cela qu'il s'agit, alors elle se trouvera elle-même à l'abri de toute offense. Mais Dianora ne peut rien faire tant qu'elle ne dispose d'aucun élément matériel à l'appui de ce qui apparaît désormais comme une certitude $^{35}$. Son intervention ne peut donc empêcher, concrètement, les agissements de Teodoro : ils en induisent ou renforcent une modalité, désormais indispensable, à savoir le caractère secret.

Cependant, le véritable rappel à l'ordre ne sera pas l'effet direct du vouloir et des dires dénonciateurs de Dianora, ni de l'action entreprise directement, et manu militari, par Lionetto. Lionetto ne retrouvera Diamante qu'un très bref instant, trop fugace pour qu'on puisse estimer qu'elle ait mis un terme au projet de Teodoro. Lionotto, après avoir pénétré dans la maison où Teodoro devait retrouver Diamante qu'il y avait fait préalablement conduire, a été arrêté pour violation de domicile et enlèvement. Le bruit court même de sa condamnation à mort, et ce bruit parvient aux oreilles de Teodoro, tout comme Arrigo (le compère de Teodoro) apprend la condamnation à mort de son fils Panfilo. Dès cet instant, les

\footnotetext{
${ }^{33}$ VA, p. 49.

${ }^{34}$ Dianora a pardonné : «C'est là un péché que la jeunesse excuse. » VA, p. 37.

${ }^{35}$ Elle ne pourra lui faire de reproches que fondés, donc dans l'après-coup : «- C'est cela l'exemple que tu donnes à ton fils. Je ne sais pas ce qui me retient de me jeter sur toi et de t'arracher les yeux de mes propres mains. »VA, p. 72 (acte V, 3).
} 
deux vieillards abandonnent leur complicité dans le mal pour assumer pleinement leur rôle de pères et pour remédier au tort qu'ils ont eux-mêmes causé. La mort possible de leurs enfants est le pire prix de leur entreprise, qui se trouve, de fait, condamnée. Le projet de séduction que Lionetto ne pouvait révéler à tous, sous peine de jeter le discrédit sur son propre nom en faisant éclater le déshonneur de son père, voici maintenant que ce sera pour lui la seule excuse qu'il pourra fournir à la violation de domicile et à l'enlèvement d'une personne, fût-elle consentante. Tout le monde saura donc la mauvaise action de Teodoro; de ce fait, il devra renoncer définitivement à Diamante, et, même si aucun mal n'a été réellement commis, chacun pourra constater un début de réalisation si ce n'est une réalisation presque accomplie. L'exposition des deux fils au risque de mort est donnée pour certaine, même si au fond une telle peine paraît sans proportion avec le délit qui leur est imputé. Mais c'est sous cette forme que la nouvelle parvient aux pères, et c'est comme telle qu'elle est reçue par eux. Une telle sanction rompt brutalement l'euphorie générale, elle met un terme à la fête du carnaval, et elle amène un dur retour au réel - le réel de l'interdit - au sein même d'une période de licence rituelle. Masqués ou non, sous couvert de carnaval ou réellement, les deux jeunes tombent sous le coup de la loi. Les pères, heureusement, et même tout à fait logiquement (mais selon une logique qui ne pouvait leur apparaître) n'auront pas à intervenir, puisqu'on apprend que les jeunes ayant expliqué en détail leur aventure, ont convaincu de leur innocence le commissaire, et ce à double titre : il n'y a eu aucune effraction, puisque Panfilo a introduit des amis à lui dans la maison de son père; il n'y a pas non plus d'enlèvement puisque Diamante ne demandait rien d'autre que de retrouver Lionetto. Le délit (doublement qualifié : enlèvement et séquestration) existait plutôt avant, et il était l'œuvre de Teodoro. Le retour à l'ordre advient donc de façon médiate, par l'entremise d'actions fortuites et non concertées au regard de l'action en cours engagée par d'autres acteurs sociaux (les témoins d'une effraction supposée telle, les forces de l'ordre...). Tout cela met fin au trouble qu'avait introduit la période de carnaval. Mais le retour à l'ordre véritable, définitif, proviendra de la fin d'un désordre antérieur à celui du carnaval, antérieur même au « désordre » qui avait contraint Lionetto à s'expatrier un certain temps, un désordre profond que le temps avait recouvert, à savoir le rapt de la sœur de Panfilo. Cette sœur revient sous les traits de Diamante précisément qui, fille d'Arrigo, ne saurait susciter la convoitise purement sensuelle de Teodoro. L'union de Lionetto et de Diamante met fin au désordre apparent et réel qu'était le quasi-inceste constitué par cette relation désirée de façon unilatérale par Teodoro ; car il s'agit bien d'une situation d'inceste transposée, l'ami du père n'étant qu'un substitut du père lui-même. On peut à ce point saisir la valeur symbolique du désordre que constituait l'anomalie sociale d'un vieil homme marié désirant enlever à son fils celle qui attend de lui un enfant. Le contrôle social s'est doublé ici d'une impossibilité sociale, d'une impossibilité radicale ; et il a mis à jour la nature gravement subversive de tout désordre, de toute dérogation à ce qui apparaît comme la norme ou l'ensemble des habitudes et des pratiques, en laissant percevoir le lien pouvant conduire de la simple infraction à l'inceste quasi réalisé. Le contrôle social est ainsi fondé à s'exercer, comme ce qui empêche la violation de la loi fondamentale de toute société, en même temps que la pièce théâtrale 
donne corps par une réalisation fictive, c'est-à-dire symbolique, au fantasme et de transgression de l'interdit fondamental.

\section{BIBLIOGRAPHIE}

\section{Textes}

GIANNOTTI, D. (1967), Il vecchio amoroso, in BoRSELlinO, N. (a cura di), Commedie del Cinquecento, vol 1, Milano, Feltrinelli, 1967, pp. 1-84.

GiannotTi, D. (2010), Opere Politiche e Letterarie di Donato Giannotti, Volume 2, Nabu Press.

GiANNOTTI, D. (1850), Opere politiche e letterarie, collazionate sui manoscritti e annotate da F. L. Polidori, precedute da un discorso di A. Vannucci, Firenze, Le Monnier, 2 vol., que l'on peut consulter en ligne sur le site Archive.org <http://www.archive.org/details/operepolitichee00giangoog> <http://www.archive.org/details/operepolitichee01 giangoog>

\section{Ouvrages critiques}

PADOAN, G. (2009), L' avventura della commedia rinascimentale, Piccin-Nuova Libraria, page 127 et page 197.

\section{Articles}

MARCONI S. (2000), Giannotti Donato in Dizionario Biografico degli Italiani, vol. 54, Roma, Istituto della Enciclopedia Italiana.

RADCLIFF D. (1974), Conflit des générations et structure comique dans « Il vecchio amoroso » de D. G., in Revue des études italiennes, XX, pp. 219-237.

\section{Articles accessibles en ligne}

KLEM, L., La commedia del Cinquecento nella dialettica tra l'immagine d'un mondo reale e la stilizzazione teatrale. Quattro esempi. $<$ http://italiensk.au.dk/fileadmin/www.italiensk.au.dk/publikationer/atti_tutto_ formato_pdf.pdf\#page=106>

OLSEN, M. (1995), Goldoni et le drame bourgeois. <http://diggy.ruc.dk/bitstream/1800/3704/1/Olsen_Goldoni.pdf>

PICQUET, T. (2000), L'humour et ses limites : Donato Giannotti, Milesia, in Italies, numéro 4, «Humour, ironie, impertinence », Revues.org. 22 déc. 2009. <http://italies.revues.org/2306> 